\title{
Research on combined kitchenware design based on general design principles
}

\author{
Yuhong $\mathrm{Wei}^{{ }^{1 *}}$, Canqun $\mathrm{He}^{1}$, Jiawei Tang ${ }^{1}$ and $\mathrm{Xiaoyu} \mathrm{Xu}^{1}$ \\ ${ }^{1}$ College of Mechanical and Electrical Engineering, Hohai University, Changzhou, Jiangsu Province, 213022, China
}

\begin{abstract}
Based on the reflection on the working environment of kitchen and the current situation of kitchenware design, the paper cares for special groups and summarizes the relationships and laws between human-kitchenware-kitchen from the perspective of various users. It introduces the general design concept into the field of kitchenware design, and put forward the design principles of modern kitchenware. Finally, the paper explains the specific application of design principles by analyzing the general combined kitchenware designed by ourselves. It reflects the importance of applying ergonomics and general design principles.
\end{abstract}

\section{Introduction}

The creation and use of tools give full play to human capabilities in the work, effectively distinguishing people from other creatures. With the continuous improvement of people's requirements and expectations for life, the research on the operability and innovation of tools has gradually deepened, and the functions of tools have been continuously refined, simplified and enhanced. Kitchenware is a typical representative of such tools.

Although there are many styles of kitchenware with complete functions and easy operation on the domestic market, the design and manufacture are not mature enough now[1]. Most of the kitchenware has the characteristics of a single function, single applicable population, and being difficult to info clerk.

Nowadays, people pay more and more attention to the general, functional, safe and user-friendly design of products. The development of kitchenware should also pay more attention to the combination of functions, general operation and human-oriented design, so that operators, including the disability, can have a better experience[2].

\section{Analysis of the law of man machine environment system in application scenarios}

\subsection{Analysis of operators}

2.1.1 Characteristics of operators. With the development of social economy, the trend of population aging is becoming more and more obvious, and the family structure has also changed a lot. The modern family structure is mainly divided into first-generation households, second-generation households, multigeneration households, and new-type households[3]. The new families include empty nest families, left-behind families, and intergenerational families and so on.

According to the characteristics of the people who undertake household chores in different family structures, contemporary families can be divided into young families, middle-aged families and elderly families. Young families include young people living alone, young couples who do not live with their parents, etc. Most of these people have the characteristics of limb coordination, fast adaptability, sloppy operation. Middle-aged families include middle-aged couples living with their children or parents. Most of these people are skilled in operation, but often tired of housework. Messy kitchen utensils and kitchen may cause negative emotions. The elderly families include empty-nest elderly people, intergenerational families, left-behind families, etc. Most of these people operate slowly and have limited strength but desire their own independence.

2.1.2 Analysis of operational obstacles / difficulties. Obstacles and difficulties in the operation of the three types of people can be inferred based on the characteristics of operators.

Young people often spill and waste due to unskilled operation, but seldom fail to understand the operating methods or adapt the operation physically. Middle-aged people often find that they can't find the kitchen utensils, the work table is messy, and they make mistakes due to fatigue. Elderly people often do not know how to use new kitchen utensils or have poor flexibility, insufficient strength and decreasing coordination.

\footnotetext{
${ }^{*}$ Corresponding author's e-mail: 1102560387@qq.com
} 
In addition, special populations, including people with disabilities and those with hidden disabilities, are prone to operating disorders[4]. The common problems are the decrease of hand flexibility, insufficient strength, and the inability of hands to cooperate, which are reflected in the difficulty of fixing kitchenware or food,

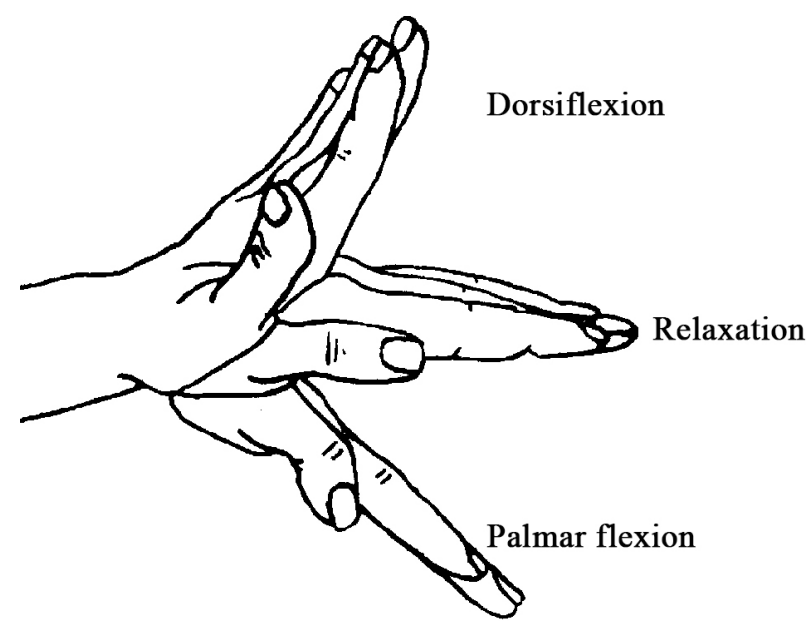

and the inability of hands to match the moving direction and mode of kitchenware.

2.1.3 Analysis of hand behavior pattern. The human hand is a relatively complicated hinge structure, but also a vulnerable part of the human body[5].

Figure 1. The state of wrist movement.

As shown in Figure 1, the physiological structure of the hand determines that the hand can only move in the vertical and horizontal planes, which are in palm curvature and dorsiflexion, radial deflection and ulnar deflection, respectively. In other relaxed states, the hand is in the middle. Therefore, the operating mode of kitchenware should ensure that the wrist is in a straight state to avoid causing wrist fatigue syndrome [6].

The usage behavior pattern of kitchenware is usually a straight-line back-and-forth movement in a holding

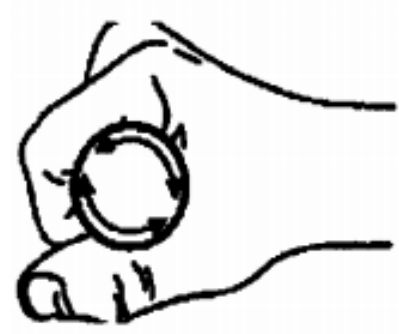

state and a continuous movement of rapid rotation of the wrist, and the motion on the vertical plane is more laborious than the motion on the horizontal plane. Therefore, in order to alleviate hand fatigue, the design of kitchenware should reduce the number of repeated wrist movements and the duration of force application, try to make the hand move on the horizontal plane, and use the arm to drive the wrist.

Figure 2. Schematic diagram of the pressure on the hands holding different shaped handles.

The shape of how to grip the handle affects the pressure distribution and blood flow of the hand muscles in the grasping state. As shown in Figure 2, when gripping a handle with a circular or triangular crosssection, a large grip force is required, and the palm is easily compressed, which may easily cause hand fatigue. The elliptical section evenly distributes the pressure on the fingers and thenar muscles, making it easy and comfortable to hold[7]. Therefore, the shape of the handle of the kitchenware should be elliptical, and the curve of the handle should fit the contour of the hand appropriately, and the diameter of the handle is between $35 \sim 45 \mathrm{~mm}$.

\subsection{Analysis of the operating environment}

The kitchen space of modern families can be roughly divided into three categories according to the layout and area of the house: fully enclosed kitchen, semi-closed kitchen and open kitchen. If space conditions permit, the cooking area, storage area, and processing area in the kitchen are usually arranged in a triangle to ensure that the operator has the best moving route[8]. However, the kitchen area of modern families is generally small, and because of the functionality of the kitchen, the cooking area and storage area are usually the most important, and the processing area is relatively small. However, there are many types of processing kitchenware, which are 
difficult to info clerk. They make the processing area more crowded and difficult to carry out activities.

In order to facilitate cleaning, the work surface in the kitchen usually adopts a smooth surface, which also brings some inconvenience to the operators: the friction between the contact surface of the kitchenware and the working table is too small and it is easy to slip, which is even more difficult for people with operational difficulties. Even they can't complete a simple process.

\section{Analysis of general combined kitchenware design}

Different from barrier-free design, general design protects the self-esteem and independence of the disabled group with a broader inclusiveness, and also provides convenience for the general population[9]. This paper puts forward the design principles of modern kitchenware by combining general design principles and the author's thinking. On this basis, a set of general combined kitchenware is designed as shown in Figure 3.
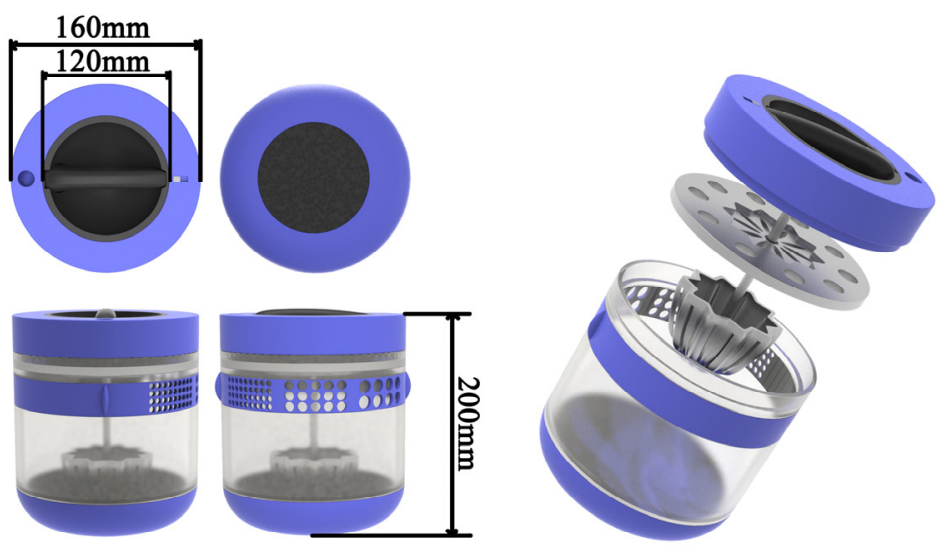

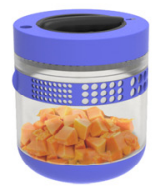

(a)

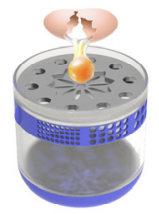

(d)

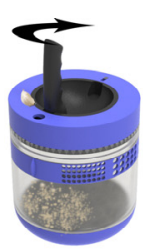

(b)

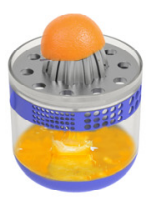

(e)

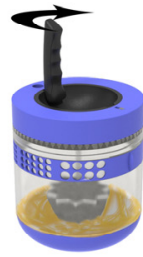

(c)

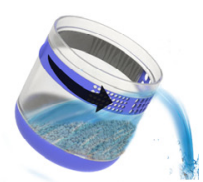

(f)

Figure 3. The display of general combined kitchenware design.

\subsection{The principle of fairness}

The principle of fairness is the most basic principle of general design, that means the design of kitchenware should meet the needs of most operators, and meet the habits and living background of most operators.

As shown in Figure 3, the functions of kitchenware should fit the daily functions required by modern kitchens and the needs of most people, avoiding extreme professionalism. The parts matching methods and operating modes of kitchen utensils should be very simple and do not require precise matching, which meets the operational ability of most people. The scale and shape of kitchenware should fit the corresponding scale of physiological structure and environment of most people. And it's refused to use any discriminatory elements.

\subsection{The principle of flexibility}

The principle of flexibility refers to the flexibility of the functions, operating methods and usage scenarios of kitchenware.

As shown in Figure 3, operators can meet different usage needs through different combinations of kitchen utensils. Kitchenware has various operating modes to meet the different features of operators. The kitchenware should be suitable for different usage scenarios, with water-proof, anti-skid, air-tight and other characteristics.

\subsection{The principle of error proof}

The principle of error proof is mainly reflected in the safety and operational compatibility of kitchenware.

As shown in Figure 3, the texture and weight of the kitchenware should be taken into consideration when choosing materials for the kitchenware. Avoid using fragile or high-density materials to prevent accidents such as scratches and injuries caused by operating errors. The rotation design of the operating rod and the anti-skid design in the bottom of kitchenware are suitable for different people who are accustomed to using left, right hand or two hands.

\subsection{The principle of low energy consumption}

The principle of low energy consumption means that the operating mode and operating process of kitchenware should be as simple, labor-saving and efficient as possible.

As shown in Figure 3, the operating process allows the operator's wrist to be in a comfortable state, and the excessive strain of the wrist is avoided by the strength of the arm. The internal gear is set to achieve coordination between input and output, improving the work efficiency.

\subsection{The principle of humanization}

The principle of humanization means that the structure, color and shape of the kitchenware should pay attention to the experience of the users. Kitchenware should be 
consciously integrated into the usage scenarios without independent existence, and anthropomorphic and interesting design can be appropriately added[10].

As shown in Figure 3, the overall shape of the kitchenware should be simple and conventional, and the parts fit each other for convenient storage. The colors are harmonious to avoid disturbing the sight. The operating mode and operating instructions of the kitchenware should be easy to understand and related to each other, with graphics, structure and other prompts, so as to facilitate the operator's understanding. For example, the indication of handle lock switch shown in Figure 4 is clear and simple, and the graphic expression is specific. Compared with the text ON / OFF, it is applicable to a wider range of people.

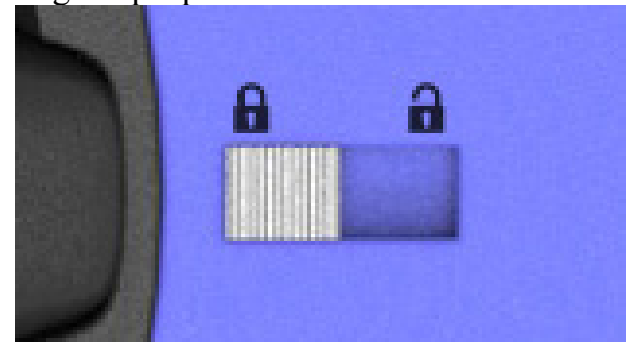

Figure 4. Switch indication.

\section{Conclusion}

The modern kitchenware embodies people's life quality and pursuit. In the design of kitchen utensils, more attention should be paid to ergonomics and design psychology in terms of function, structure, form, appearance and so on, so as to meet the requirements of general design better. This paper summarizes the deficiencies and difficulties existing in the field of kitchenware design through analyzing the different characteristics of operators and operating environments of kitchenware, and proposes five major principles of modern kitchenware design: the principle of fairness, flexibility, error proof, low energy consumption and humanization. Referring to the five principles, a new design scheme of combined kitchenware is proposed, focusing on the functional combination, innovation and generality of the kitchenware, which has a certain guiding role and reference value in the future kitchenware design. And the concept of general design can be further developed in the kitchenware design.

\section{References}

1. Liang, X.T., Zhang, A.W. (2018) The art of writing a scientific article. Architectural engineering technology and design, 12: 1356.

2. Sun, L. (2018) Development trend and design orientation of kitchen and kitchenware. Literary life, 8: $30-31$

3. Jiang, M. (2018) Research on the design of family kitchen appliances from the perspective of general design. Suzhou University, Suzhou.

4. Chen, C. (2019) Research on integrated design of kitchen products and environment based on general design. Industrial Design, 3: 48-50.

5. Ji, Q. (2018) Research on the shape optimization design of angle grinder handle based on holding mode. Shandong University of science and technology, Shandong.

6. Ding, Y.L. (2000) Ergonomics. Beijing University of Technology Press, Beijing.

7. Xia, R.L. (2013) Research on grip comfort of tool handle based on pressure distribution. Zhejiang University of technology, Hangzhou.

8. Yang, X.W, Zhang, M. (2007) Research on the Design of "Humanized" Kitchen. In: China man machine environment system engineering conference. Shenzhen. pp. 452-457.

9. Li, J.B. (2014) From barrier free design to universal design. From barrier free design to universal design. Art review, 9: 143-146.

10. He, M. (2010) Research on the design of kitchenware in the new economic era. Hebei University of science and technology, Hebei. 